\title{
Squeezed Fermions at Relativistic Heavy Ion Colliders
}

\author{
P. K. Panda ${ }^{1}$, T. Csörgö ${ }^{1,2,3,4}$, Y. Hama ${ }^{3}$, G. Krein ${ }^{1}$ and Sandra S. Padula ${ }^{1}$ \\ ${ }^{1}$ Instituto de Física Teórica, Universidade Estadual Paulista, \\ Rua Pamplona 145, 01405-900 São Paulo - SP, Brazil, \\ 2 MTA KFKI RMKI, H - 1525 Budapest 114, POB 49, Hungary, \\ ${ }^{3}$ Instituto de Física, Universidade de São Paulo, \\ C.P. 66318 - CEP 05315-970 São Paulo, Brazil \\ ${ }^{4}$ Department of Physics, Columbia University, $538 \mathrm{~W}$ 120th St, \\ New York, NY 10027
}

\begin{abstract}
Large back-to-back correlations of observable fermion - anti-fermion pairs are predicted to appear, if the mass of the fermions is modified in a thermalized medium. The back-to-back correlations of protons and anti-protons are experimentally observable in ultra-relativistic heavy ion collisions, similarly to the Andreev reflection of electrons off the boundary of a superconductor. While quantum statistics suppresses the probability of observing pairs of fermions with nearby momenta, the fermionic back-to-back correlations are positive and of similar strength to bosonic back-to-back correlations.
\end{abstract}

PACS numbers: 25.75.Gz,25.75.-q,74.80.Fp 


\section{INTRODUCTION}

In high energy heavy ion collisions, hot and dense hadronic matter is expected to be created in conditions similar to the ones in the early Universe about a few $\mu$ sec after the Big Bang. Under these conditions, strongly interacting particles may propagate with a mass that differs from the mass in the asymptotic vacuum. Recently, it has been discovered that in-medium mass-modifications induce large back-to-back correlations (BBC) among pairs of asymptotic, observable bosons [1,2]. However, it was not known before if the bosonic BBC would have an analogous effect in the fermion sector. In this Letter we show that fermion anti-fermion pairs also exhibit BBC, if they propagate with a modified mass in the medium. The physical picture is that of a system in thermodynamical equilibrium which freezes-out suddenly. The masses and other properties of the hadrons in the system are modified due to interactions and a quasi-particle description is assumed. At freeze-out the quasi-particles are converted into the free particles that will be detected. Particle-anti-particle pairs detected with momenta back-to-back give rise to a quantum-mechanical correlation due to a nonzero overlap between the in-medium states and the free states. The quantum correlation can be calculated with a Bogoliubov-Valatin transformation between a quasi-particle basis and a free-particle basis.

The bosonic BBC (bBBC) have a quantum optical analogy, namely the correlations in thermalized ensembles of two-mode squeezed states. It turns out that fermion BBC (fBBC) also have an analogy, which is the Andreev reflection, well-known in solid state physics. It refers to the scattering of electrons off the boundary of a superconductor - normal conductor junction. The reflected electrons are used to study the properties of the superconductors [3]. Our results for fBBC generalize Andreev's reflection to the case when superconductivity is suddenly switched off in the whole volume of the material, so that a junction prevails not at a given position for a long time but in the whole medium at a given instant.

Quantum statistics enhances the probability of observing pairs of bosons in similar momentum states, while it suppresses the probability of observing pairs of fermions with nearby 
momenta. In spite of such Fermi-Dirac suppression factors that appear in the evaluation of the correlation function, we find that the dominant term of the $\mathrm{fBBC}$, due to the BogoliubovValatin transformation, is positive and similar in strength to the bosonic BBC of Refs. [1,2]. The fBBC could be observed experimentally in ${ }^{197} \mathrm{Au}+{ }^{197} \mathrm{Au}$ collisions at the Relativistic Heavy Ion Collider (RHIC), which started to take data at the Brookhaven National Laboratory at $\sqrt{s}=56$ and $130 \mathrm{AGeV}$ in 2000 and which will reach its full designed energy of $\sqrt{s}=200 \mathrm{AGeV}$ in the near future.

The outline of the paper is as follows. In section II we consider the in-medium mass modification state at finite temperature. The spectra and the correlations for mass-shifted fermions are described in section III and section IV contains concluding remarks.

\section{BASIC ASSUMPTIONS}

We assume the validity of the concepts of local thermalization, hydrodynamics, and a short duration of particle emission. These concepts are in agreement with the observable single-particle spectra and two-particle correlations of pions, kaons and protons [四]. We also assume the validity of the effective Hamiltonian

$$
H=H_{0}+H_{I}
$$

where

$$
H_{0}=\int d \mathbf{x}: \bar{\psi}(\mathbf{x})(-i \boldsymbol{\gamma} \cdot \boldsymbol{\nabla}+M) \psi(\mathbf{x}):
$$

is the Hamiltonian in vacuum, and the interaction Hamiltonian $H_{I}$ describes the medium modifications and will be discussed shortly below. In Eq. (2), $M$ is the value of the proton (or in general, baryon) mass in free space, $\psi$ and $\bar{\psi}$ are the fermion field operators which satisfy the usual equal-time anti-commutation relations.

We are interested in the single-particle and two-particle invariant momentum distributions for fermions and anti-fermions: 


$$
\begin{aligned}
& N_{1}\left(\mathbf{k}_{1}\right)=\omega_{\mathbf{k}_{1}}\left\langle a_{\mathbf{k}_{1}}^{\dagger} a_{\mathbf{k}_{1}}\right\rangle, \quad \tilde{N}_{1}\left(\mathbf{k}_{1}\right)=\omega_{\mathbf{k}_{1}}\left\langle\tilde{a}_{\mathbf{k}_{1}}^{\dagger} \tilde{a}_{\mathbf{k}_{1}}\right\rangle \\
& N_{2}\left(\mathbf{k}_{1}, \mathbf{k}_{2}\right)=\omega_{\mathbf{k}_{1}} \omega_{\mathbf{k}_{2}}\left\langle a_{\mathbf{k}_{1}}^{\dagger} \tilde{a}_{\mathbf{k}_{2}}^{\dagger} \tilde{a}_{\mathbf{k}_{2}} a_{\mathbf{k}_{1}}\right\rangle .
\end{aligned}
$$

Here, $\langle\hat{O}\rangle$ denotes the expectation value of operator $\hat{O}$ in the thermalized medium, and the $a^{\dagger}, a, \tilde{a}^{\dagger}, \tilde{a}$ are creation and annihilation operators of free baryons and anti-baryons of mass $M$ and energy $\omega_{\mathbf{k}}=\sqrt{M^{2}+|\mathbf{k}|^{2}}$. These creation and annihilation operators are defined through the expansion of the baryon field operator as

$$
\psi(\mathbf{x})=\frac{1}{\sqrt{V}} \sum_{\lambda, \lambda^{\prime}, \mathbf{k}}\left(u_{\lambda, \mathbf{k}} a_{\lambda, \mathbf{k}}+v_{\lambda^{\prime},-\mathbf{k}} \tilde{a}_{\lambda^{\prime},-\mathbf{k}}^{\dagger}\right) e^{i \mathbf{k} \cdot \mathbf{x}}
$$

where $V$ is the volume of the system, $u_{\lambda, \mathbf{k}}$ and $v_{\lambda^{\prime},-\mathbf{k}}$ are Dirac spinors and the summation extends over momenta $\mathbf{k}$ and spin projections $\lambda, \lambda^{\prime}=1 / 2,-1 / 2$. The creation and annihilation operators satisfy canonical anti-commutation relations. The particle-anti-particle correlation function is defined as

$$
C_{2}\left(\mathbf{k}_{1}, \mathbf{k}_{2}\right)=\frac{N_{2}\left(\mathbf{k}_{1}, \mathbf{k}_{2}\right)}{N_{1}\left(\mathbf{k}_{1}\right) \tilde{N}_{1}\left(\mathbf{k}_{2}\right)} .
$$

As in the bosonic case [1,2], the expectation value of the four baryon operators in Eq. (田) can be calculated using Wick's theorem generalized for a locally equilibrated system, with the result

$$
\left\langle a_{\mathbf{k}_{1}}^{\dagger} \tilde{a}_{\mathbf{k}_{2}}^{\dagger} \tilde{a}_{\mathbf{k}_{2}} a_{\mathbf{k}_{1}}\right\rangle=\left\langle a_{\mathbf{k}_{1}}^{\dagger} a_{\mathbf{k}_{1}}\right\rangle\left\langle\tilde{a}_{\mathbf{k}_{2}}^{\dagger} \tilde{a}_{\mathbf{k}_{2}}\right\rangle-\left\langle a_{\mathbf{k}_{1}}^{\dagger} \tilde{a}_{\mathbf{k}_{2}}\right\rangle\left\langle\tilde{a}_{\mathbf{k}_{2}}^{\dagger} a_{\mathbf{k}_{1}}\right\rangle+\left\langle a_{\mathbf{k}_{1}}^{\dagger} \tilde{a}_{\mathbf{k}_{2}}^{\dagger}\right\rangle\left\langle\tilde{a}_{\mathbf{k}_{2}} a_{\mathbf{k}_{1}}\right\rangle .
$$

The minus sign in the above equation is due to Fermi statistics. The expectation values involving $a_{\mathbf{k}_{1}}^{\dagger} a_{\mathbf{k}_{2}}$ and $\tilde{a}_{\mathbf{k}_{1}}^{\dagger} \tilde{a}_{\mathbf{k}_{2}}$ give rise to the chaotic amplitudes, while $a_{\mathbf{k}_{1}} \tilde{a}_{\mathbf{k}_{2}}$ gives rise to the "squeezed" amplitude, defined as

$$
\begin{aligned}
& G_{c}\left(\mathbf{k}_{1}, \mathbf{k}_{2}\right)=\sqrt{\omega_{\mathbf{k}_{1}} \omega_{\mathbf{k}_{2}}}\left\langle a_{\mathbf{k}_{1}}^{\dagger} a_{\mathbf{k}_{2}}\right\rangle, \quad \tilde{G}_{c}\left(\mathbf{k}_{1}, \mathbf{k}_{2}\right)=\sqrt{\omega_{\mathbf{k}_{1}} \omega_{\mathbf{k}_{2}}}\left\langle\tilde{a}_{\mathbf{k}_{1}}^{\dagger} \tilde{a}_{\mathbf{k}_{2}}\right\rangle \\
& G_{s}\left(\mathbf{k}_{1}, \mathbf{k}_{2}\right)=\sqrt{\omega_{\mathbf{k}_{1}} \omega_{\mathbf{k}_{2}}}\left\langle a_{\mathbf{k}_{1}} \tilde{a}_{\mathbf{k}_{2}}\right\rangle .
\end{aligned}
$$

The chaotic amplitudes are non-vanishing for identical type of fermions and anti-fermions, while the squeezed amplitude is non-vanishing only for particle-anti-particle pairs. We will 
show in the following that, as in the case of bosons [1,2], the squeezed $G_{s}\left(\mathbf{k}_{1}, \mathbf{k}_{2}\right)$ can be considerably large if the in-medium masses of the baryons are different from their free-space values.

In order to evaluate the thermal averages above we model the system as a globally thermalized gas of quasi-particles (quasi-baryons). The density matrix for such a system is given not in terms of the free-space creation and annihilation operators $a^{\dagger}, \tilde{a}, \cdots$, but by a new set that we denote by $b^{\dagger}, \tilde{b}, \cdots$. They are of course different because of medium effects, described by the interacting Hamiltonian in Eq. (1). While it is the $a$-quanta that are observed as asymptotic states, it is the $b$ quanta that are thermalized in the medium.

In a quasi-particle description of the system, the medium effects are taken into account through a self-energy function. For a spin-1/2 particle under the influence of mean fields in a many-body system, one can write its self-energy function as

$$
\Sigma=\Sigma^{s}+\gamma^{0} \Sigma^{0}+\gamma^{i} \Sigma^{i}
$$

This function can be determined from a detailed calculation based on, for example, the Walecka or the Zimányi - Moszkowski models [5,6]. Calculations in the context of such models show that $\Sigma^{0}$ is weakly momentum dependent and $\Sigma^{i}$ is very small (see for example Ref. [6] for detailed numerical results). It is well known that a tensor contribution may also occur, however, in applications for nuclear matter and finite nuclei this additional component is negligible, and will not be considered here. Therefore, we assume $\Sigma^{0}$ independent of momentum, and neglect $\Sigma^{i}$. We denote the scalar component of the self-energy as $\Sigma^{s}(\mathbf{k})=$ $\Delta M(\mathbf{k})$. Now, for a locally thermalized system, the role of $\Sigma^{0}$ is to shift the chemical potential, i.e.

$$
\mu_{*}=\mu-\Sigma^{0}
$$

As we present our results as functions of the net baryon density, the value of $\Sigma^{0}$ (or the difference between $\mu$ and $\mu_{*}$ ) and its $\mu$ dependence need not be specified. More specifically, since we specify the total baryon density, we invert the expression of the density in terms of $\mu_{*}$ 
to obtain the baryon and anti-baryon Fermi-Dirac distributions (see Eq. (17) below). In this way, the value of $\Sigma^{0}$ and its dependence on $\mu$ are not needed. With these approximations, the effective Hamiltonian of Eq. (1) thus describes a system of quasi-particles with a momentumdependent mass $M_{*}(\mathbf{k})=M-\Delta M(\mathbf{k})$.

In the context of the model we just described, the free-space and in-medium creation and annihilation operators are related through a fermionic Bogoliubov-Valatin transformation [7]

$$
\left(\begin{array}{c}
a_{\lambda, \mathbf{k}} \\
\tilde{a}_{\lambda^{\prime},-\mathbf{k}}^{\dagger}
\end{array}\right)=\left(\begin{array}{ccc}
c_{\mathbf{k}} & \frac{f_{\mathbf{k}}}{\left|f_{\mathbf{k}}\right|} s_{\mathbf{k}} A \\
-\frac{f_{\mathbf{k}}^{*}}{\left|f_{\mathbf{k}}\right|} s_{\mathbf{k}}^{*} A^{\dagger} & c_{\mathbf{k}}^{*}
\end{array}\right)\left(\begin{array}{c}
b_{\lambda, \mathbf{k}} \\
\tilde{b}_{\lambda^{\prime},-\mathbf{k}}^{\dagger}
\end{array}\right),
$$

where $c_{\mathbf{k}}=\cos f_{\mathbf{k}}, s_{\mathbf{k}}=\sin f_{\mathbf{k}}, A$ is a $2 \times 2$ matrix with elements $A_{\lambda, \lambda^{\prime}}=\chi_{\lambda}^{\dagger} \sigma \cdot \hat{\mathbf{k}} \tilde{\chi}_{\lambda^{\prime}}$, where $\hat{\mathbf{k}}=\mathbf{k} /|\mathbf{k}|, \chi$ is a Pauli spinor and $\tilde{\chi}=-i \sigma^{2} \chi$. Here, $f_{\mathbf{k}}$ is the squeezing function. In the present case, $f_{\mathbf{k}}$ is real and we will therefore drop the complex-conjugate notation in what follows. The effective Hamiltonian is brought to a diagonal form in the basis of the $b$ quanta when the squeezing function $f_{\mathbf{k}}$ is related to the mass shift $\Delta M$ as [ [B],

$$
\tan \left(2 f_{\mathbf{k}}\right)=-\frac{|\mathbf{k}| \Delta M(\mathbf{k})}{\omega_{\mathbf{k}}^{2}-M \Delta M(\mathbf{k})}
$$

With this, the thermal averages are then evaluated with the following density matrix operator

$$
\hat{\rho}=\frac{1}{Z} \exp \left(-\frac{1}{T} \frac{V}{(2 \pi)^{3}} \int d \mathbf{k}\left[\left(\Omega_{\mathbf{k}}-\mu_{*}\right) b_{\lambda \mathbf{k}}^{\dagger} b_{\lambda, \mathbf{k}}+\left(\Omega_{\mathbf{k}}+\mu_{*}\right) \tilde{b}_{\lambda \mathbf{k}}^{\dagger} \tilde{b}_{\lambda, \mathbf{k}}\right]\right)
$$

where $V$ is the volume of the system, $T$ the temperature, and $\Omega_{\mathbf{k}}=\left(\mathbf{k}^{2}+M_{*}{ }^{2}\right)^{1 / 2}$ is the quasi-particle energy. $Z$ is the trace of the exponential factor in Eq. (14).

The evaluation of the thermal averages in Eqs. (8) and (9) is very easy and the results are

$$
\begin{aligned}
\left\langle a_{\lambda, \mathbf{k}}^{\dagger} a_{\lambda^{\prime}, \mathbf{k}^{\prime}}\right\rangle & =\left(c_{\mathbf{k}}^{2} n_{\mathbf{k}}+s_{\mathbf{k}}^{2}\left(1-\tilde{n}_{\mathbf{k}}\right)\right) \delta_{\lambda \lambda^{\prime}} \delta_{\mathbf{k k}^{\prime}}, \\
\left\langle\tilde{a}_{\lambda,-\mathbf{k}} a_{\lambda^{\prime}, \mathbf{k}^{\prime}},\right\rangle & =\left(1-\tilde{n}_{\mathbf{k}}-n_{\mathbf{k}}\right) c_{\mathbf{k}} s_{\mathbf{k}}(A)_{\lambda \lambda^{\prime}} \delta_{\mathbf{k k}^{\prime}},
\end{aligned}
$$

where 


$$
n_{\mathbf{k}}=\frac{1}{\exp \left[\left(\Omega_{\mathbf{k}}-\mu_{*}\right) / T\right]+1}, \quad \tilde{n}_{\mathbf{k}}=\frac{1}{\exp \left[\left(\Omega_{\mathbf{k}}+\mu_{*}\right) / T\right]+1} .
$$

The terms $\left\langle\tilde{a}_{\lambda, \mathbf{k}}^{\dagger} a_{\lambda^{\prime}, \mathbf{k}^{\prime}}\right\rangle$ and $\left\langle a_{\lambda, \mathbf{k}}^{\dagger} \tilde{a}_{\lambda^{\prime}, \mathbf{k}^{\prime}}\right\rangle$ in Eq. (7) vanish because they involve expectation values of two baryon annihilation operators, or two anti-baryon creation operators, or one baryon and one anti-baryon operators.

\section{SPECTRA AND CORRELATIONS FOR MASS-SHIFTED FERMIONS}

If a thermal gas of $b$ fermions freezes out suddenly at temperature $T$, the observed single particle and anti-particle spectrum are given by

$$
\begin{aligned}
& N_{1}(\mathbf{k})=\frac{V}{(2 \pi)^{3}} \omega_{\mathbf{k}}\left[c_{\mathbf{k}}^{2} n_{\mathbf{k}}+s_{\mathbf{k}}^{2}\left(1-\tilde{n}_{\mathbf{k}}\right)\right], \\
& \tilde{N}_{1}(\mathbf{k})=\frac{V}{(2 \pi)^{3}} \omega_{\mathbf{k}}\left[s_{\mathbf{k}}^{2}\left(1-n_{\mathbf{k}}\right)+c_{\mathbf{k}}^{2} \tilde{n}_{\mathbf{k}}\right] .
\end{aligned}
$$

Note that the single-particle (anti-particle) spectrum includes a squeezed contribution. While the thermal part of the spectrum falls off exponentially for large values of $|\mathbf{k}|$, the squeezed contribution falls off only as a power of $|\mathbf{k}|$, because of the term proportional to $s_{\mathbf{k}}^{2}$ in Eq. (18).

For such a homogeneous system, the net baryon density $\rho_{B}$, the chaotic, and the squeezed amplitudes can be written from Eqs. (15) and (16). They are given by

$$
\begin{aligned}
\rho_{B} & =\frac{g}{V} \sum_{\mathbf{k}}\left(n_{\mathbf{k}}-\tilde{n}_{\mathbf{k}}\right), \\
G_{c}\left(\mathbf{k}_{1}, \mathbf{k}_{2}\right) & =\frac{V}{(2 \pi)^{3}} \omega_{\mathbf{k}_{1}}\left[c_{\mathbf{k}_{1}}^{2} n_{\mathbf{k}_{1}}+s_{\mathbf{k}_{1}}^{2}\left(1-\tilde{n}_{\mathbf{k}_{1}}\right)\right] \delta_{\mathbf{k}_{1}, \mathbf{k}_{2}}, \\
\tilde{G}_{c}\left(\mathbf{k}_{1}, \mathbf{k}_{2}\right) & =\frac{V}{(2 \pi)^{3}} \omega_{\mathbf{k}_{1}}\left[s_{\mathbf{k}_{1}}^{2}\left(1-n_{\mathbf{k}_{1}}\right)+c_{\mathbf{k}_{1}}^{2} \tilde{n}_{\mathbf{k}_{1}}\right] \delta_{\mathbf{k}_{1}, \mathbf{k}_{2}}, \\
G_{s}\left(\mathbf{k}_{1}, \mathbf{k}_{2}\right) & =\frac{V}{(2 \pi)^{3}} \omega_{\mathbf{k}_{1}}\left[\left(1-n_{\mathbf{k}_{1}}-\tilde{n}_{\mathbf{k}_{1}}\right) c_{\mathbf{k}_{1}} s_{\mathbf{k}_{1}} A^{\dagger}\right] \delta_{\mathbf{k}_{1},-\mathbf{k}_{2}} .
\end{aligned}
$$

There are two kinds of fermionic two-particle correlation functions. Similarly to the bosonic case [2], let us denote by $C_{2}^{(++)}\left(\mathbf{k}_{1}, \mathbf{k}_{2}\right)$ the case when the two particles are identical fermions, and by $C_{2}^{(+-)}\left(\mathbf{k}_{1}, \mathbf{k}_{2}\right)$ when particle 1 is a fermion and 2 is an anti-fermion. The correlation 
functions $C_{2}^{(--)}\left(\mathbf{k}_{1}, \mathbf{k}_{2}\right)$ and $C_{2}^{(-+)}\left(\mathbf{k}_{1}, \mathbf{k}_{2}\right)$ can be obtained from the $(++)$ and $(+-)$ correlations by a trivial exchange of particle and anti-particle labels. For an infinite, homogeneous thermalized medium, these correlation functions are non-trivial only for identical or back-toback momenta, $\mathbf{k}_{2}= \pm \mathbf{k}_{1}$. We find that $C_{2}^{(++)}(\mathbf{k}, \mathbf{k})=C_{2}^{(--)}(\mathbf{k}, \mathbf{k})=0$, the canonical value of the Fermi-Dirac correlation function, reflecting the anti-correlation of identical fermions, due to the Pauli exclusion principle.

The $\mathrm{BBC}$ for fermion - anti-fermion pairs given by Eq. (6), reads as

$$
\begin{aligned}
C_{2}^{(+-)}(\mathbf{k},-\mathbf{k}) & =1+\frac{\left|G_{s}(\mathbf{k},-\mathbf{k})\right|^{2}}{G_{c}(\mathbf{k}, \mathbf{k}) \tilde{G}_{c}(-\mathbf{k},-\mathbf{k})} \\
& =1+\frac{\left(1-n_{\mathbf{k}}-\tilde{n}_{\mathbf{k}}\right)^{2}\left(c_{\mathbf{k}} s_{\mathbf{k}}\right)^{2}}{\left[c_{\mathbf{k}}^{2} n_{\mathbf{k}}+s_{\mathbf{k}}^{2}\left(1-\tilde{n}_{\mathbf{k}}\right)\right]\left[c_{\mathbf{k}}^{2} \tilde{n}_{\mathbf{k}}+s_{\mathbf{k}}^{2}\left(1-n_{\mathbf{k}}\right)\right]} .
\end{aligned}
$$

From this equation it follows that the fermionic BBC are unlimited from above. For sufficiently large values of $|\mathbf{k}|$, the Fermi-Dirac distribution $n_{\mathbf{k}}$ falls exponentially, while $s_{\mathbf{k}}$ decreases only as a power-law. Hence, for sufficiently large values of $|\mathbf{k}|$, the fBBC diverge as in the case of bosonic BBC, as $C_{2}^{(+-)}(\mathbf{k},-\mathbf{k}) \propto 1+1 / s_{\mathbf{k}}^{2} \rightarrow \infty$. This divergence happens for small values of the mass-shift and for large values of $\mathbf{k}$ in both the fermionic and the bosonic cases.

We next discuss numerical results. We use momentum-independent in-medium masses, as one would obtain in a mean-field approximation in model calculations of the sort discussed in Refs. [5:6]. There is no intrinsic difficulty in using momentum-dependent self-energies but a commitment to a specific model is then necessary. Since in this initial study we are more interested in the qualitative effects, rather than in precise, quantitative predictions, we simply use typical values for $M_{*}$ as obtained in mean-field calculations. In Fig. 1 we show the fBBC for $p \bar{p}$ pairs as a function of the in-medium mass $M_{*}=M-\Delta M$, for three illustrative values of the net baryonic density $\rho_{B}$ : the normal nuclear matter density, one tenth of the nuclear matter density, and $\rho_{B}=0$. This last value of $\rho_{B}$ corresponds to a baryon-free region, as expected to be formed at RHIC. We also show in this figure the corresponding result for bBBC of $\phi$ mesons. Following Ref. [2], a finite time suppression factor is used to model a more gradual freeze-out. A detailed derivation for a gradual exponential freeze-out 
leads to a factor of the form [2],

$$
|\tilde{F}(\mathbf{k})|^{2}=\frac{1}{\left[1+\left(2 \Delta t \omega_{\mathbf{k}}\right)^{2}\right]},
$$

which multiplies $C_{2}^{(+-)}(k, k)-1$, the non-trivial part of the back-to-back correlation function. We use $\Delta t=2 \mathrm{fm} / \mathrm{s}$, as in Ref. [2]. We observe that the fBBC are strongly enhanced when the net baryonic density decreases, and that the shape of fBBC for $\rho_{B}=0$ is rather similar to bBBC. For a fixed temperature, as we increase the net baryon density obviously we have an excess of baryon over anti-baryons. On the other hand, the fBBC will be larger for approximately equal baryon and anti-baryon densities and therefore the effect will be enhanced as we approach zero net baryon density. Our result shows that for a baryon free region, the $\mathrm{BBCs}$ are approximately independent of the bosonic or the fermionic nature of the particles. In addition, the $\mathrm{fBBC}$ are not only positive, as in the bosonic case, but they are also of the same order of magnitude as the corresponding bBBC.

The expected magnitude of the effect is illustrated in Fig. 2 for two, typical momenta of thermal-looking proton spectrum in $\mathrm{Pb}+\mathrm{Pb}$ collisions at CERN SPS [9], for $|\mathbf{k}|=500$ $\mathrm{MeV}$ and for $|\mathbf{k}|=800 \mathrm{MeV}$. As in Fig. 1, the finite time suppression factor of Eq. (24) is used. Fig. 2 indicates that the fBBC is strongly enhanced for increasing momentum $|\mathbf{k}|$. Fig. 2 also highlights that the magnitude of the $\mathrm{fBBC}$ is greatly enhanced as the net baryon density decreases from normal nuclear density to a vanishing value. Similarly to the bBBC case, the strength of $\mathrm{fBBC}$ is very sensitive to the shape of the freeze-out distribution, as well as to the value of the parameter $\Delta t$, the particle freeze-out duration, as discussed in Ref. [2]. Note that in the limit of infinitely slow freeze-out, $\Delta t \rightarrow \infty$, both fBBC and bBBC vanish.

It is particularly interesting to compare the BBC discussed above in Eq. (23) with Eq. (19) of Ref. [2] for bosonic case as

$$
\begin{aligned}
C_{2}^{(+-)}(\mathbf{k},-\mathbf{k}) & =1+\frac{\left(1+n_{\mathbf{k}}^{b}+n_{\mathbf{k}}^{b}\right)^{2}\left(c_{\mathbf{k}}^{b} s_{-\mathbf{k}}^{b}\right)^{2}}{\left[\left(c_{\mathbf{k}}^{b}\right)^{2} n_{\mathbf{k}}^{b}+\left(s_{-\mathbf{k}}^{b}\right)^{2}\left(1+n_{\mathbf{k}}^{b}\right)\right]\left[\left(c_{\mathbf{k}}^{b}\right)^{2} n_{\mathbf{k}}^{b}+\left(s_{\mathbf{k}}^{b}\right)^{2}\left(1+n_{\mathbf{k}}^{b}\right)\right]}, \\
n_{\mathbf{k}}^{b} & =\frac{1}{\exp \left(\Omega_{\mathbf{k}}^{b} / T\right)-1} .
\end{aligned}
$$


Note, however, that the symbols $s_{\mathbf{k}}$ and $c_{\mathbf{k}}$ are related to $\sin f_{\mathbf{k}}$ and $\cos f_{\mathbf{k}}$, as defined by Eq.(13) in the fermionic case, while in the bosonic case, these symbols mean $s_{\mathbf{k}}^{b}=\sinh f_{\mathbf{k}}^{b}$ and $c_{\mathbf{k} B}^{b}=\cosh f_{\mathbf{k}}^{b}$, where $f_{\mathbf{k}}^{b}=\frac{1}{2} \log \left(\omega_{\mathbf{k}} / \Omega_{\mathbf{k}}\right)$, as given by Eq. (11) of Ref. [2] , is also real. It follows that the BBC diverge with increasing $|\mathbf{k}|$ as the inverse of the single-particle spectra, both in the fermionic and bosonic cases.

\section{CONCLUSION}

BBC stands for Back-to-Back Correlations of particle and anti-particle pairs. These correlations appear if in-medium interactions lead to the modification of hadronic masses in the medium. The origin of these back-to-back correlations is an entirely quantum effect, related to the propagation of particle fields through a space-like boundary surface between the medium and the asymptotic region. The strength of these correlations can be unlimitedly large, and the shape of the BBC is similar for fermions and bosons. Deep mathematical and physical reasons are behind these similarities. A sudden freeze-out of thermalized medium-

modified quanta to asymptotic fields is described by a bosonic or a fermionic Bogoliubov transformation. Although these transformations are canonical, they connect Fock spaces that become unitarily inequivalent in the infinite volume limit and the state of the medium corresponds to strongly correlated, squeezed particle-anti-particle states of the asymptotic quanta.

At large values of momenta, the similarity of fermionic $\mathrm{BBC}$ to bosonic $\mathrm{BBC}$ reflects a symmetry of the decaying medium to observable boson - anti-boson and fermion - antifermion pairs. This effect vanishes in case of exactly zero in-medium mass-modification, but for large values of $|\mathbf{k}|$ and small values of mass-shifts, BBC can be very large and should be observable if the freeze-out process of the medium modified quanta is sufficiently sudden, for example if the duration of particle freeze-out is within $2 \mathrm{fm} / \mathrm{c}$.

In this Letter, we have extended the concept of back-to-back correlations to an important new domain of broad experimental and theoretical interest. As a reduction of the net baryon 
density by a factor of 10 increases dramatically the magnitude of the effect, the almost baryon-free mid-rapidity region of $A u+A u$ collisions at RHIC seems to be an ideal place to find the back-to-back correlations of proton - anti-proton or $\Lambda-\bar{\Lambda}$ pairs experimentally.

\section{ACKNOWLEDGMENTS}

We thank M. Asakawa, M. Gyulassy and G. Zimányi for useful comments and suggestions during the completion of the manuscript. T. Csörgő is grateful to Y. Hama and S. S. Padula for creating an inspiring working atmosphere during his visit in São Paulo and to M. Gyulassy for the kind hospitality at the Columbia University. This research has been supported in part by CNPq and FAPESP grants 98/2249-4, 99/08544-0, 99/09113-3, by the Hungarian OTKA T025435, T029158, the US - Hungarian Joint Fund MAKA 652/1998 and the NWO-

OTKA grant N025186, by the US Department of Energy grants DE - FG02 - 93ER40764, DE - FG02 - 92 - ER40699, DE - AC02 - 76 - CH00016 and by a Bolyai Fellowship. 


\section{REFERENCES}

[1] M. Asakawa and T. Csörgö, hep-ph/9612331, Heavy Ion Physics 4, (1996) 233; M. Asakawa and T. Csörgó, quant-ph/9708006, in proc. Strong and Electroweak Matter'97 Conference, Eger, Hungary, May 1997. (World Scientific, Singapore, 1998, F. Csikor and Z. Fodor eds.) p. 332.

[2] M. Asakawa, T. Csörgő and M. Gyulassy, Phys. Rev. Lett. 83, (1999) 4013.

[3] A. F. Andreev. Sov. Phys. JETP 19, (1964) 1228; see e.g. H. Hoppe, U. Zülicke and G. Schön, Phys. Rev. Lett. 84, (2000) 1804; J. Torrès and T. Martin, Eur. J. Phys. B12 (1999) 319.

[4] A. Ster, T. Csörgő and B. Lörstad, Nucl. Phys. A661, (1999) 419c.

[5] J.D. Walecka, Ann. Phys. (N.Y.) 83, (1974) 491; J. Zimányi and S. Moszkowski, Phys. Rev. C. 42, (1990) 1416.

[6] A. Bouyssy, J.-F. Mathiot, N.V. Giai and S. Marcos Phys. Rev. C 36, (1987) 380.

[7] A. Mishra, P.K. Panda, S. Schramm, J. Reinhardt, W. Greiner, Phys. Rev. C 56, (1997) 1380.

[8] A.L. Fetter J.D. Walecka, Quantum Theory of Many-Particle Systems (McGraw-Hill, NewYork, 1971).

[9] H. Appelshauser et al, NA49 Collaboration, Phys. Rev. Lett. 82, (1999) 2471. 


\section{FIGURES}

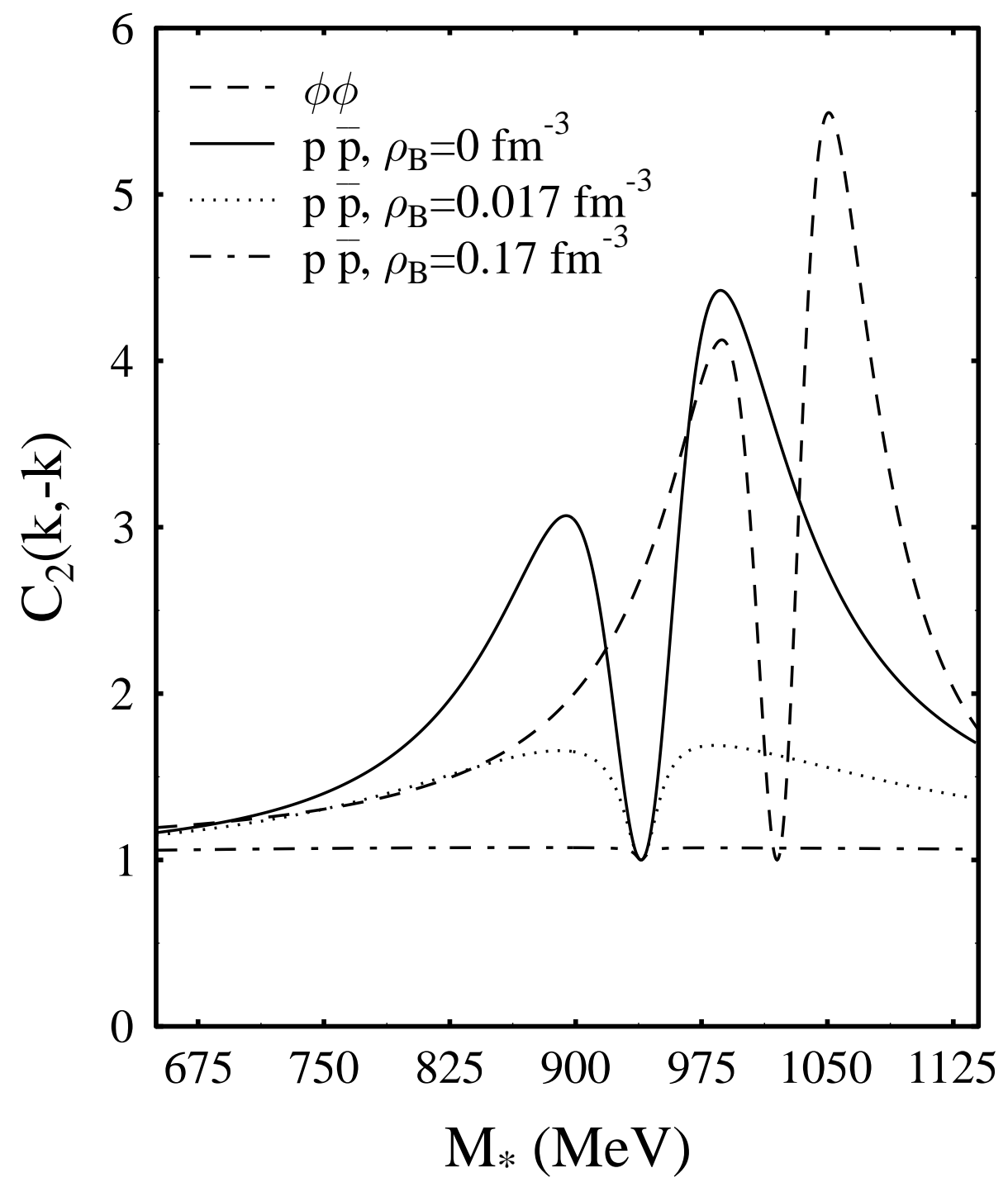

FIG. 1. Back-to-back correlations of proton - anti-proton pairs and $\phi$-meson pairs, for $T=140 \mathrm{MeV}, \Delta t=2 \mathrm{fm} / \mathrm{c}$ and $|\mathbf{k}|=800 \mathrm{MeV} / \mathrm{c}$. 

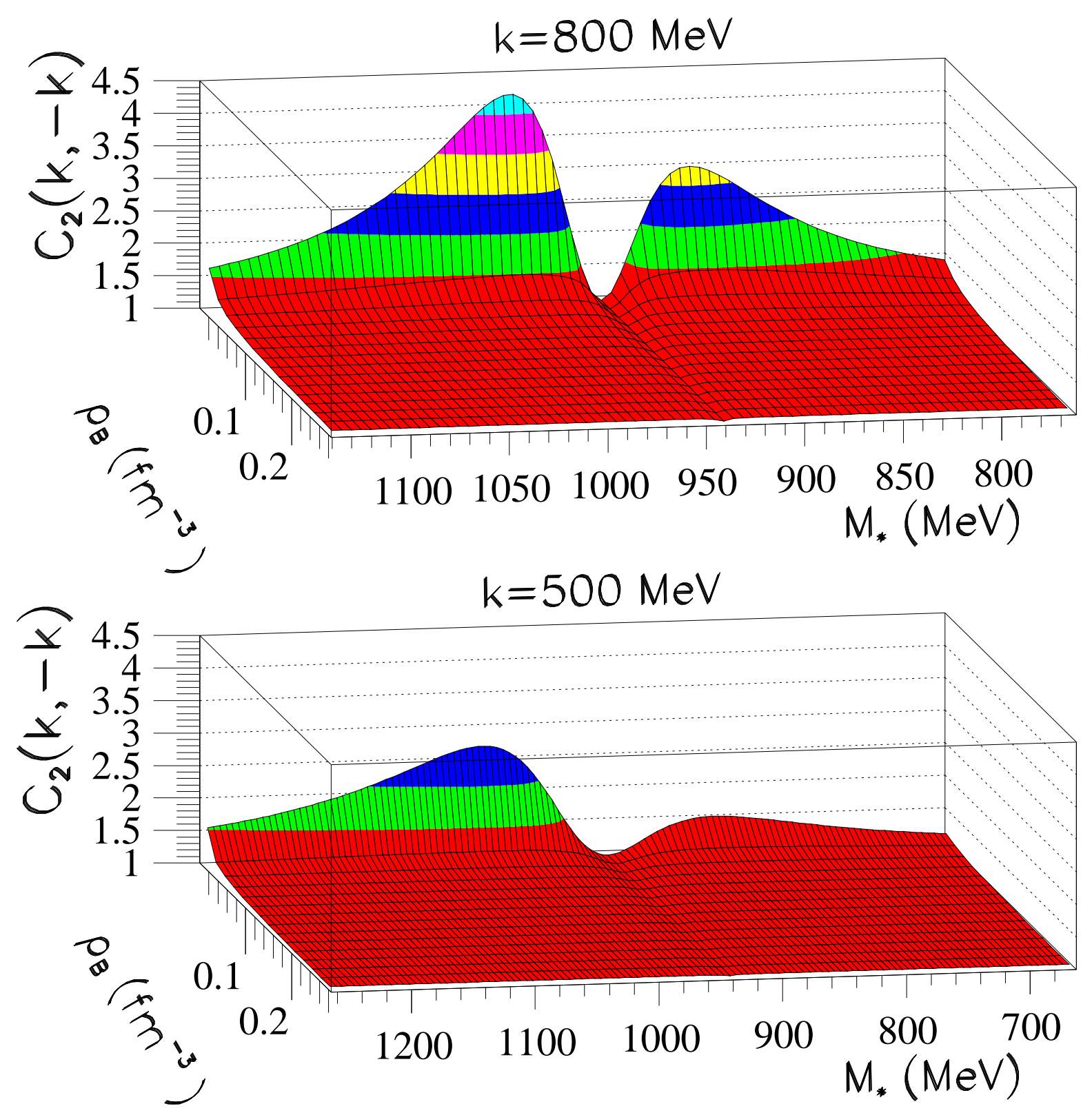

FIG. 2. Dependence of the fBBC on the in-medium modified proton mass, $M_{*}$, and on the net baryon density, $\rho_{B}$, for $T=140 \mathrm{MeV}, \Delta t=2 \mathrm{fm} / \mathrm{c}$ and two typical values of the momentum $|\mathbf{k}|$. The fBBC strongly increases with decreasing net baryon density and increasing values of $|\mathbf{k}|$. 